\title{
SARS-CoV-2 infection in children requiring hospitalization: the experience of Navarra, Spain
}

\author{
Laura Moreno-Galarraga ${ }^{1,2,3}$ (1) - María Urretavizcaya-Martínez ${ }^{4} \cdot J^{\prime}$ osune Alegría Echauri ${ }^{5} \cdot$ Marcos García Howard $^{4}$. \\ Eva Ruperez García ${ }^{4}$ · Sergio Aguilera-Albesa ${ }^{2,6}$ • Valentín Alzina de Aguilar ${ }^{7,8}$ • Mercedes Herranz Aguirre Men $^{2,4}$
}

Received: 30 June 2020 / Accepted: 10 September 2020 / Published online: 28 September 2020

(c) Children's Hospital, Zhejiang University School of Medicine 2020

\begin{abstract}
Background Coronavirus disease 2019 in children, or pediatric COVID-19, initially was described as an acute respiratory syndrome similar to the adult presentation but with less severe manifestations.

Methods We describe the clinical characteristics, disease presentation, treatments and outcomes of all pediatric cases with COVID-19 admitted to the reference hospitals in Navarra, Spain during the first wave of the COVID-19 outbreak (February-May 2020).

Results We found a low number of hospitalized cases in infants and children compared to adults with a hospitalization ratio of 1:180. Most of these hospitalized cases did not suffer from severe disease. Over $80 \%$ of infections reported household contacts, and the mother was the known-contact in $83 \%$ of these cases. $72 \%$ of hospitalized cases were previously healthy children. We describe how symptoms in pediatric cases are nonspecific and how COVID-19 can be presented with a wide range and variety of symptoms. Respiratory symptoms are not always present, and severe neurological and cardiac features can occur in previously healthy children.

Conclusion Epidemiological description and case reports will be key to a better recognition and to adequate treatment of pediatric patients with COVID-19.
\end{abstract}

Keywords Coronavirus disease $2019 \cdot$ Hospitalization · Severe acute respiratory syndrome coronavirus 2

\section{Introduction}

Since severe acute respiratory syndrome coronavirus 2 (SARS-CoV-2) emerged as the etiological pathogen responsible of a cluster of severe pneumonia [1], this coronavirus has rapidly spread worldwide, causing a global pandemic [2]. The World Health Organization (WHO) initially named

Laura Moreno-Galarraga

laura.moreno.galarraga@cfnavarra.es;

lauramoreno11@yahoo.es

1 Pediatric Pulmonology, Department of Pediatrics, CHN, Complejo Hospitalario de Navarra, Servicio Navarro de Salud, C/Irunlarrea 3, 31080 Pamplona, Spain

2 IdiSNA, Navarra Institute for Health Research, Pamplona, Spain

3 Department of Preventive Medicine and Public Health, School of Medicine, University of Navarra, Pamplona, Spain

4 Department of Pediatrics, CHN, Complejo Hospitalario de Navarra, Servicio Navarro de Salud, Pamplona, Spain this new virus as the 2019 novel coronavirus (2019-nCoV), but in February, the coronavirus study group of the International Committee on Taxonomy of Viruses renamed it as SARS-CoV-2 and also named the disease caused by it as coronavirus disease 2019 (COVID-19). On January 30th, the WHO declared the outbreak of COVID-19 a public health emergency [3], and just 3 months later (May 1st 2020), 
3,175,207 confirmed cases were reported and 224,162 deaths were related to COVID-19 worldwide [4]. Even though the disease has affected hundreds of thousands of people, data on clinical presentation in children remain scarce.

COVID-19 cases are mostly diagnosed among adults, with few pediatric cases [5], and first data indicated that children accounted for only a $1-5 \%$ of total COVID-19 cases. Publications and case reports suggested that clinical presentation on children was similar to the acute respiratory syndrome seen in adults, but milder, generally presenting with low respiratory infections symptoms and with pediatric deaths being extremely rare [6-10].

Nevertheless, pediatricians from different Europeans countries have recently reported severe cases of COVID-19 in children, and how children with SARS-CoV-2 infection can either present from totally asymptomatic to a wide range of clinical symptoms. Digestive manifestations (including abdominal pain, vomiting or diarrhea) seem to be frequent in pediatric patients, and in the last weeks, some cases of severe SARS-CoV-2 infection have been reported in previously healthy children, with high inflammatory markers and acute cardiac failure, suggestive of myocarditis [7, 11].

Many infectious diseases affect children in a different way than adults, and it is only by recognizing and understanding these differences that we can shed light on this novel infection and the host response to it. These differences may be keys to finding better treatments and preventive strategies. We believe that case reports and pediatric case series are needed to better understand how this novel coronavirus can affect pediatric patients, to determine which children are at higher risk, and to recognize red flags of severe COVID-19 cases.

The objective of this article is to describe the wide range of clinical presentations seen among hospitalized pediatric patients with COVID-19 in Navarra, Spain from February 1st to May 31st, 2020.

\section{Methods}

We retrospectively retrieved epidemiological information and clinical data for all pediatric patients (aged 0-15 years) hospitalized with laboratory confirmed COVID-19 in Navarra, during the first outbreak. Navarra is a province in northeast Spain with 660,887 inhabitants, located close to the Pyrenees along the border with France. Navarra has a current pediatric population of 101,000 children [12]. It has five general hospitals with pediatric emergency departments that admit pediatric patients. However, during the first wave of the COVID-19 pandemic, only the two tertiary hospitals located in the capital, Pamplona, admitted severe pediatric cases (Complejo Hospitalario de Navarra: CHN and Clínica Universidad de Navarra:
CUN). Navarra has a very developed public healthcare system, and medical records are centralized in a unified computerized medical history.

The first COVID-19 case in Spain was reported on January 31st, and when cases in Madrid increased significantly, the Spanish Government declared the State of Emergency on March 13th. Severe measures, including home confinement and strict social isolation, were imposed to control the spread of SARS-CoV-2. The first adult case with COVID-19 was hospitalized in Navarra on February 28th, and the first pediatric case was admitted on March 19th. The peak of the general CHN-hospitalization curve occurred during the last week of March, reaching a maximum of 461 hospitalized cases per day. By May 2020, a total of 4848 confirmed SARS-CoV-2 cases, 1977 hospitalized patients and 458 deaths had been registered in Navarra. Initial cases were associated with travelers from countries with COVID-19 epidemic, but since March 12th, the stage of "community transmission" was reached in Navarra. On May 2020, the curve of Navarra's CHN-hospitalized adult cases with COVID-19 reflected a mono-peak pattern in a late descending phase (Fig. 1). Epidemiological investigation included the study of the route of transmission, assessing travel history, residence in community transmission areas or close contact with family member with COVID-19.

SARS-CoV-2 is transmitted from person to person through respiratory droplets and close contact $[1,6,13]$ with rapid community transmission and frequent nosocomial infections. The mean incubation period is about 5 days (1-14 days) [10]. The most common symptoms among adults are fever, cough and respiratory distress [5-7]. According to the Spanish health department protocol for SARS-CoV-2 infection diagnosis, real-time polymerase chain reaction (RT-PCR) was performed at local reference laboratories. Quantitative RT-PCR was considered positive if respiratory samples were positive by both two targets genes: open reading frame lab gene and nucleocapsid protein gene (ORFlab and $N$ ). A less than 35-cycle threshold value was defined as a positive test. During the first wave of the COVID-19 outbreak, two types of SARS-CoV-2 detection assays were available at CHN, the RT-PCR assay based on the presence and amplification of viral RNA and antibody testing, which detects the presence of antibodies against SARS-CoV-2 based on enzyme-linked immunosorbent assays. Samples for proofreading PCR (PR-PCR) assays were taken from the upper respiratory tract (nasopharyngeal and oropharyngeal swabs), and all tests were performed according to the European Commission Guidelines on COVID-19 in vitro diagnostic tests. PR-PCR was performed on admission for diagnosis, but no PR-PCR was performed at discharge and patients were told to be isolated at home for 14 days after hospital discharge. 

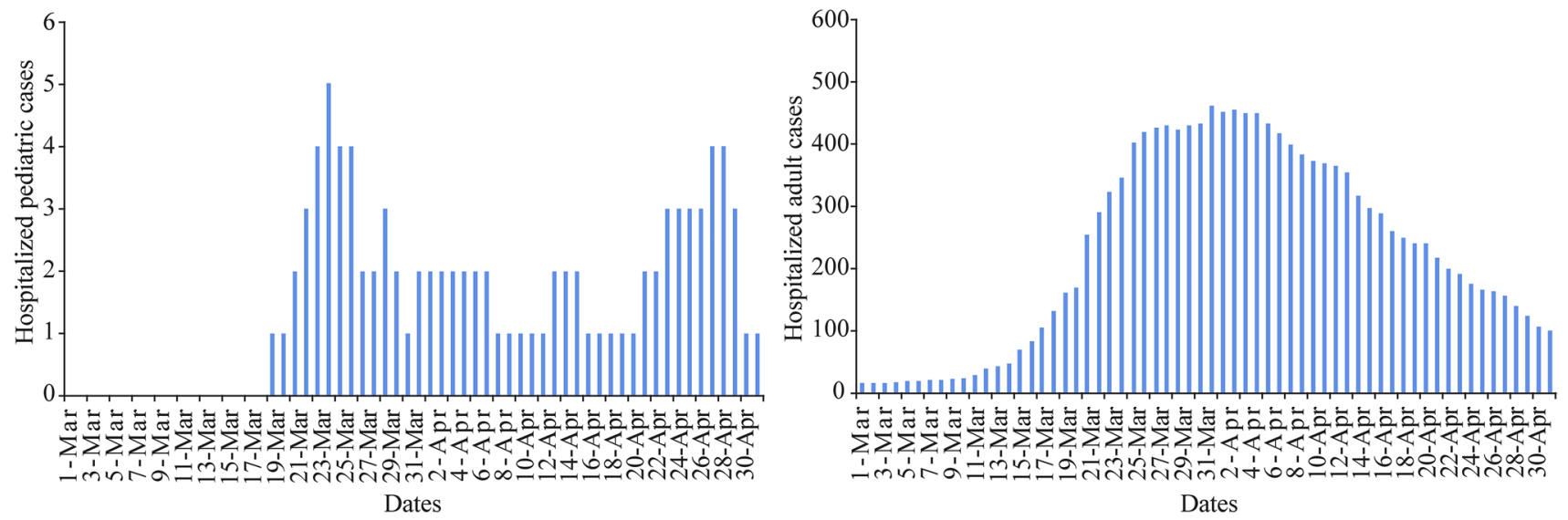

Fig. 1 Graphic representation of adult and pediatric hospitalized cases with COVID-19 in Navarre Hospital Complex during February 1st 2020May 1st 2020, Navarra, Spain

If RT-PCR was positive or if serologic tests for SARSCoV-2 were positive [immunoglobulin (Ig) G, IgM or both], a patient was diagnosed as a confirmed COVID-19 case. If diagnosis was made using clinical criteria (epidemiological exposure and fever or suggestive symptoms), a patient was diagnosed as a possible COVID-19 case.

In Navarra, during the first wave of the pandemic, not all pediatric confirmed COVID-19 cases were hospitalized. Only severe cases or high-risk patients were admitted into the hospital. Also, owing to limited resources, not all patients with suggestive clinical criteria were tested and therefore confirmed as COVID-19 cases.

This is a multi-center observational cohort study using data from tertiary reference hospitals in Navarra between February and May 2020. Confirmed and hospitalized pediatric cases were recruited for this study and are detailed in this case-series report. On May 2020, the Ethics Committee of Navarra approved the study in pediatric population (PI_2020/38). Informed consent was obtained from parents or guardians to include patients in a patient register, and permission to use their clinical data for publishing was requested following local Ethics Committee guides. During the COVID-19 pandemic, oral consent waived forms were accepted to minimize the risk of transmission during the informed consent process. A study team member obtained oral consent for all patients included in this study from a parent or legally authorized representative. Oral consent was obtained during children hospitalization, and oral authorization was recorded in patient personal medical history. A doctor, in the presence of a nurse or a study team member, explained information to the parents. Medical records review, access to laboratory results, and personal interviews with each patient's responsible pediatrician were performed to collect better case information. Data on demographics, exposure history, medical history, clinical presentations, treatment and laboratory tests were collected. Possible COVID-19 cases were not included. SARS-CoV-2 viral testing was positive in all included cases. We defined severe cases as those with severe clinical presentations (such as shock, sepsis, respiratory failure or altered state of consciousness) or with severe alterations in complementary tests (such as significant coagulation dysfunction, myocardial injury or refractive-hypoxia). Cases that needed admission or monitoring in an intensive care unit (ICU) also were considered to be severe COVID-19 cases.

\section{Results}

Between March 1st and May 31st, a total of 309 children (aged 0-15 years) were diagnosed as "confirmed COVID19 cases" in Navarra. Among them, 91 (29.5\%) patients were diagnosed by a positive RT-PCR and the rest by a positive serologic test. Most (96.5\%) COVID-19 confirmed pediatric cases were followed up as outpatients by their local pediatrician, and many children with suggestive COVID-19 symptoms were diagnosed as "possible cases" at their local Health Care Centers, where SARSCoV-2 detection assays were not available.

Between February and May 2020, during the first wave of the COVID-19 outbreak in Navarra, a total of 18 children with suggestive SARS-CoV-2 infection were admitted into the pediatric COVID-19 ward. Eleven cases were confirmed (ten patients with positive RT-PCR and one by positive serologic test). RT-PCR for SARS-CoV-2 was performed on all patients within 12 hours after consulting in the emergency room. One case (case 9) also had fecal samples tested for the RNA of SARS-CoV-2, but the test was negative. Of all "confirmed COVID-19" pediatric 
cases in Navarra, 3.5\% (11/309) were hospitalized. We here report the epidemiological and clinical features of the 11 confirmed COVID-19 hospitalized children.

\section{Basal characteristics of cases}

Table 1 shows the epidemiological and clinical characteristics of the 11 confirmed pediatric patients with COVID-19, including the main symptoms and the exposure history. The patients' ages ranged from 1 month to 15 years $(6.37 \pm 5.87$ years $)$. Seven patients were female. Five patients were Caucasian, three Arabic, two Latin American and one Gypsy. Two patients (cases 4 and 5) were overweight or obese. Case 2 had a congenital respiratory malformation (left congenital lung hypoplasia). Case 4 had a congenital cardiac disease (corrected complete atrio-ventricular canal defect, with residual mitral insufficiency) and Down syndrome, and case 8 had a nephrotic syndrome. Eight (72\%) cases were previously healthy children. All cases were correctly immunized according to their ages and local vaccination schedule, and three patients (cases 2, 4 and 8) also had $2020 \mathrm{flu}$ vaccination. Seven children had a confirmed contact with a COVID-19 case. Most of these (86\%) were household contacts, and the mother was the source of infection in $83 \%$ of these cases. In four patients, the source of infection remained unknown, and none of them had a travel history to epidemic areas.

\section{Main symptoms of COVID-19}

The reasons for consultation in the emergency room were diverse. Seven (64\%) patients referred fever, 5 (45\%) cough, $3(27 \%)$ referred respiratory distress, 2 (18\%) rhinorrhea and $1(9 \%)$ sore throat. Six (54\%) patients did not refer any kind of respiratory symptoms at all. Fever generally resolved 3-4 days after onset. The highest fever reported was $40.2^{\circ} \mathrm{C}$, and 4 (36\%) patients did not report any fever. Eight (72\%) patients referred abdominal symptoms, three referred vomits, three diarrheas and three abdominal pain. None of the children had other symptoms frequently referred by adults, such as dyspnea, muscle ache, headache, or anosmia.

\section{Hospitalization}

In the $\mathrm{CHN}$, the hospitalization ratio of children to adult was 1:180. No pediatric deaths were reported. Among Navarra's pediatric population, hospitalization rate was 1:10,000 children. Among the confirmed pediatric COVID-19 cases, the hospitalization rate was $1: 28$. Duration of pediatric hospitalization ranged from 3 to 12 days. The median hospitalstay was 6.72 days. Most (72\%) pediatric patients were discharged in less than 1 week. Only one patient (case 9) needed admission to a pediatric ICU, due to an acute myocarditis and shock. ICU stay was 9 days, and he was then discharged and followed up in an outpatients' clinic.

\section{Laboratory tests: blood-routine + coagulation function + inflammatory markers}

Blood tests were performed at admission in all children and were repeated during follow-up in nine cases. At admission, mean values and standard deviation (SD) were the following (data expressed in mean $\pm \mathrm{SD}$ ): hemoglobin $13.1 \pm 1.3 \mathrm{~g} / \mathrm{dL}$, white blood cells $9675 \pm 6900 / \mathrm{mm}^{3}$, neutrophils $5226 \pm 6508 / \mathrm{mm}^{3}$, lymphocytes $3116 \pm 2650.8 / \mathrm{mm}^{3}$, platelets $250,392 \pm 143,127 / \mu \mathrm{L}$, alanine aminotransferase $24.5 \pm 15.2 \mathrm{U} / \mathrm{L}$, aspartate aminotransferase $39 \pm 11.9 \mathrm{U} / \mathrm{L}$, bilirubin $1.94 \pm 3.7 \mathrm{mg} / \mathrm{dL}$, ferritin $106 \pm 79 \mu \mathrm{g} / \mathrm{L}$, fibrinogen $545 \pm 182 \mathrm{mg} / \mathrm{dL}$, prothrombin time $14 \pm 3.3$ seconds, partial thromboplastin time $33.5 \pm 6.3$ seconds. Three patients had neutrophilia ( $>7500$ neutrophils $/ \mu \mathrm{L}$ ) at admission, with all patients having normal neutrophils at discharge. High white blood cell count or neutrophilia was not associated with higher elevation of other inflammatory markers or worse clinical evolution. At admission, C-reactive protein (CRP) was $46.1 \pm 43.1 \mathrm{mg} / \mathrm{L}$ and procalcitonin was $0.39 \pm 0.5 \mathrm{ng} / \mathrm{mL}$. At discharge, CRP was $17.1 \pm 19.4 \mathrm{mg} / \mathrm{L}$ and procalcitonin was $0.18 \pm 0.14 \mathrm{ng} / \mathrm{mL}$. D-dimer was $1499 \pm 1599 \mathrm{ngFEU} / \mathrm{mL}$, with 7/11 patients showing elevated D-dimer values. All patients who had high-density lipoprotein tested had high values $(328.7 \pm 104 \mathrm{U} / \mathrm{L})$. Glucose was normal in all patients $(99 \pm 36 \mathrm{mg} / \mathrm{dL})$. Cardiac enzymes were evaluated in seven patients and were elevated in three of those: mean troponin $40.4 \pm 70.6 \mathrm{pg} / \mathrm{mL}$, with a maximum value of $261 \mathrm{pg} / \mathrm{mL}$ in case 9 . Interleukin-6 was measured only in case 9 and was elevated (558 pg/ $\mathrm{mL}$; normal value $<4$ ). Laboratory tests found that $81 \%$ of pediatric hospitalized patients had leukocytosis, $23 \%$ had neutrophilia, $63 \%$ had lymphopenia, and $91 \%$ of cases associated elevation of inflammatory markers. Lymphopenia was more frequent in older patients: mean age of patients with lymphopenia at admission was $12.84 \pm 2.26$ years vs. mean age of all patients was $6.37 \pm 5.87$ years $(P<0.001)$. Table 2 shows laboratory tests for each case, and normal reference values. Lumbar puncture was performed in case 7 (an infant with non-febrile seizures), and cerebrospinal fluid (CSF) analysis for cells, glucose and protein were normal. The PCR results for herpes virus, enterovirus, and bacterial culture were negative in CSF. Blood cultures were negative in all patients, urine cultures were negative except case 6 (Escherichia coli urinary tract infection) and case 2 had a positive Campylobacter-PCR in feces. PCR for influenza, respiratory syncytial viral and most common respiratory virus was performed and was negative in all patients, except 


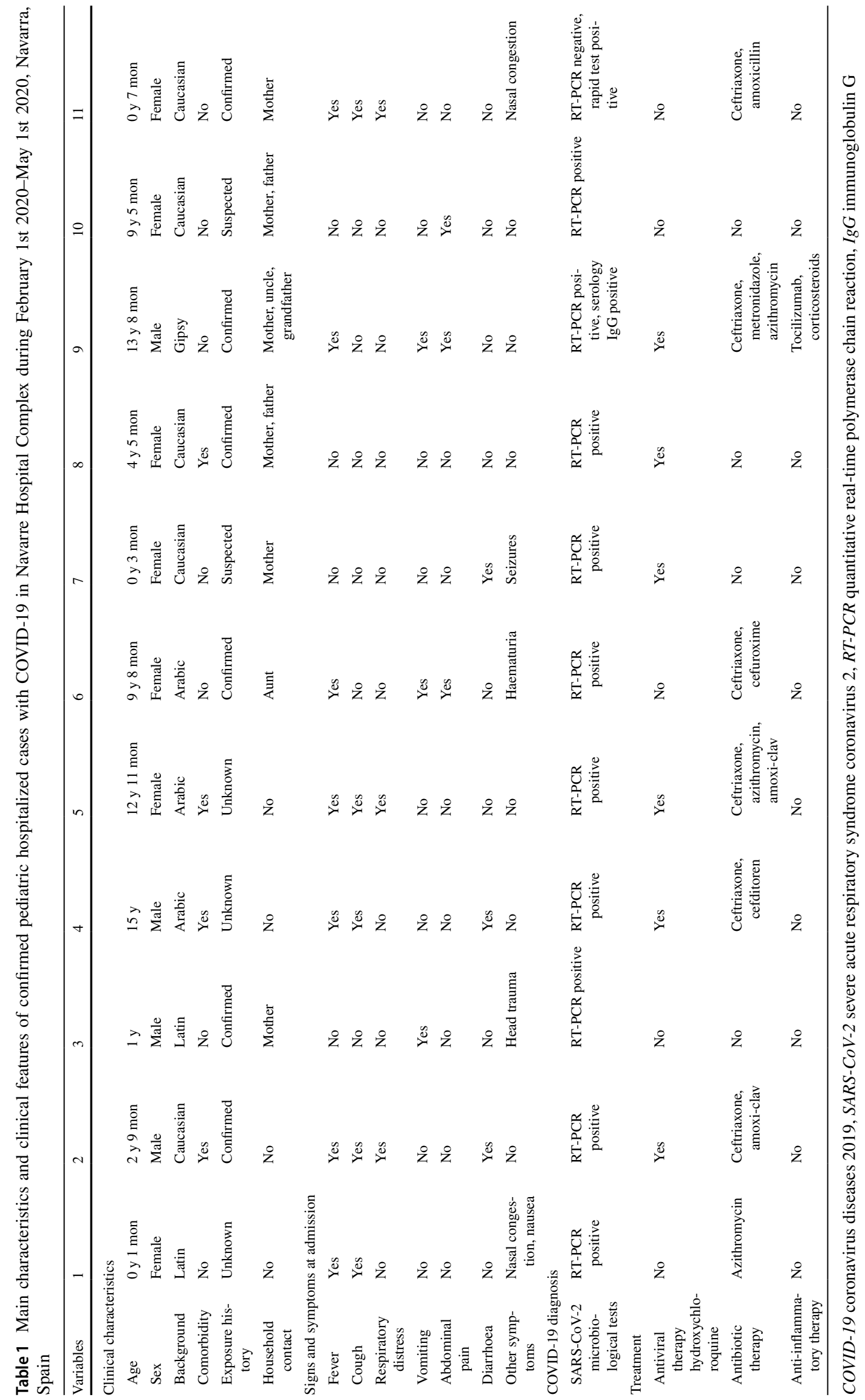


case 2 who showed a co-infection with rhinovirus, enterovirus and parainfluenzae.

\section{Complementary tests}

(1) Electrocardiogram was performed in six (55\%) patients, if hydroxychloroquine was evaluated as a possible treatment or if cardiac enzymes were elevated. All electrocardiograms were normal, except case 9 who had ventricular extra-systoles; (2) chest X-rays were performed in all patients (Table 2). They revealed bilateral interstitial infiltrates or grinding glass in four cases and focal pneumonia-consolidation in one case. No pleural effusion or complicated pneumonias were seen. Seven (63\%) chest X-rays were normal; (3) abdominal ultrasound was performed in two cases, showing unspecific ileo-colic inflammation in case 9; (4) others: echocardiography, thoracoabdominal-computed tomography (CT) scan and cardiac-magnetic resonance imaging (MRI), were performed in case 9 , with results compatible with acute myocarditis.

\section{Therapeutic strategies}

All patients received symptomatic treatment with antipyretics or analgesic when needed. Fluid therapy was administered in two patients. One patient needed oxygen therapy, two patients used inhaled bronchodilators (salbutamol), and seven patients with suspected bacterial co-infection were treated with empirical antibiotic therapy, with a mean duration of 10 days. Ceftriaxone was the preferred antibiotic (85\%), as its regimen (once daily) minimizes nurse's exposure. Anticoagulant prophylaxis was used only in case 9. Hydroxychloroquine was used in six patients, dosage was $6.5 \mathrm{mg} / \mathrm{kg} / \mathrm{day}$. In case 9 (acute myocarditis), inotropic drugs (adrenaline and milrinone), intravenous injection of corticosteroids and tocilizumab were used. Remdesivir was requested, but due to Spanish Health Government policies, this drug was not available initially and when received, case 9 had already initiated recovery and the drug was not used. Invasive mechanical ventilation or other respiratory support was not used in any of the hospitalized pediatric cases with COVID-19.

\section{Description of severe pediatric cases with COVID-19}

Based on the criteria of severe disease, three children were considered as severe cases in our study. These cases were previously healthy children and did not differ from mild cases in age, sex, laboratory test or clinical presentation. Severe cases at our hospital, presented with higher levels of D-dimer values at admission, but no significant association was found between severe and non-severe cases.
Case 1 was a 1-month-old baby girl that had coagulopathy with very high D-dimer values (7037 ngFEU/mL) at admission. Following pediatric protocols, prophylactic anticoagulant treatment was not initiated; the patient remained asymptomatic and values resolved spontaneously. The D-dimer values were less than $2000 \mathrm{ngFEU} / \mathrm{mL}$ at discharge, and normal at follow-up.

Case 7 was an infant with afebrile seizures. A previously healthy, 2-month-old girl was admitted for recurrent convulsions without fever. The mother informed that 3 days before onset of seizures, the infant had presented low fever and respiratory symptoms. Upon admission, patient blood tests were normal, except high ferritin value. RT-PCR test was positive for SARS-CoV-2. Additional testing for other infectious agents was negative. Chest-X-ray, brain-CT scan and cerebral MRI did not reveal abnormalities; CSF analysis was normal. Levetiracetam was started but hydroxychloroquine was added owing to the persistence of seizures in a COVID-19 setting. The patient then recovered and had no new seizures. Inflammatory markers were normal at followup, and the patient was diagnosed with benign infantile seizure associated with COVID-19.

Case 9 was a 13-year-old healthy male that presented in the emergency room complaining of abdominal pain and high fever, with associated palms skin lesions. He was admitted and initially tested negative for SARS-CoV-2 (two RT-PCR results were negative). The epidemiological situation (mother with positive RT-PCR five weeks before) and the blood results (severe lymphopenia, increased CRP, ferritin, inflammatory markers and coagulopathy) made us suspect a COVID-19-related illness. As a result, serologies were performed (with positive SARS-CoV-2 IgG) and a repeated RT-PCR was positive. On day 2 , the patient developed hemodynamic instability (hypotension and tachycardia $85 / 50 \mathrm{mmHg}, 140 \mathrm{bpm}$ ). In the next hours, control blood test showed a hyperimmune response, with raised IL-6 $(558 \mathrm{pg} / \mathrm{mL})$, increased troponin and elevation in other inflammatory markers. The patient developed intermittent chest-pain, electrocardiogram showed cardiac arrhythmia with monomorphic ventricular extrasystoles, and echocardiography revealed a reduced left ventricular systolic function. Cardiac pathology was suspected, and the patient was transferred to CUN-ICU. Vasoactive drugs were started, and cardiac-MRI confirmed acute myocarditis. No respiratory symptoms were present, and chest X-ray and thorax-CT scan were normal. Patient was treated with hydroxychloroquine, tocilizumab, empiric antibiotics and standard treatment for acute myocarditis (gammaglobulins, foscarnet and intravenous injection of steroids). Blood tests one month after discharge were completely normal, and cardiac function was fully recovered. The patient recovered without sequelae. 


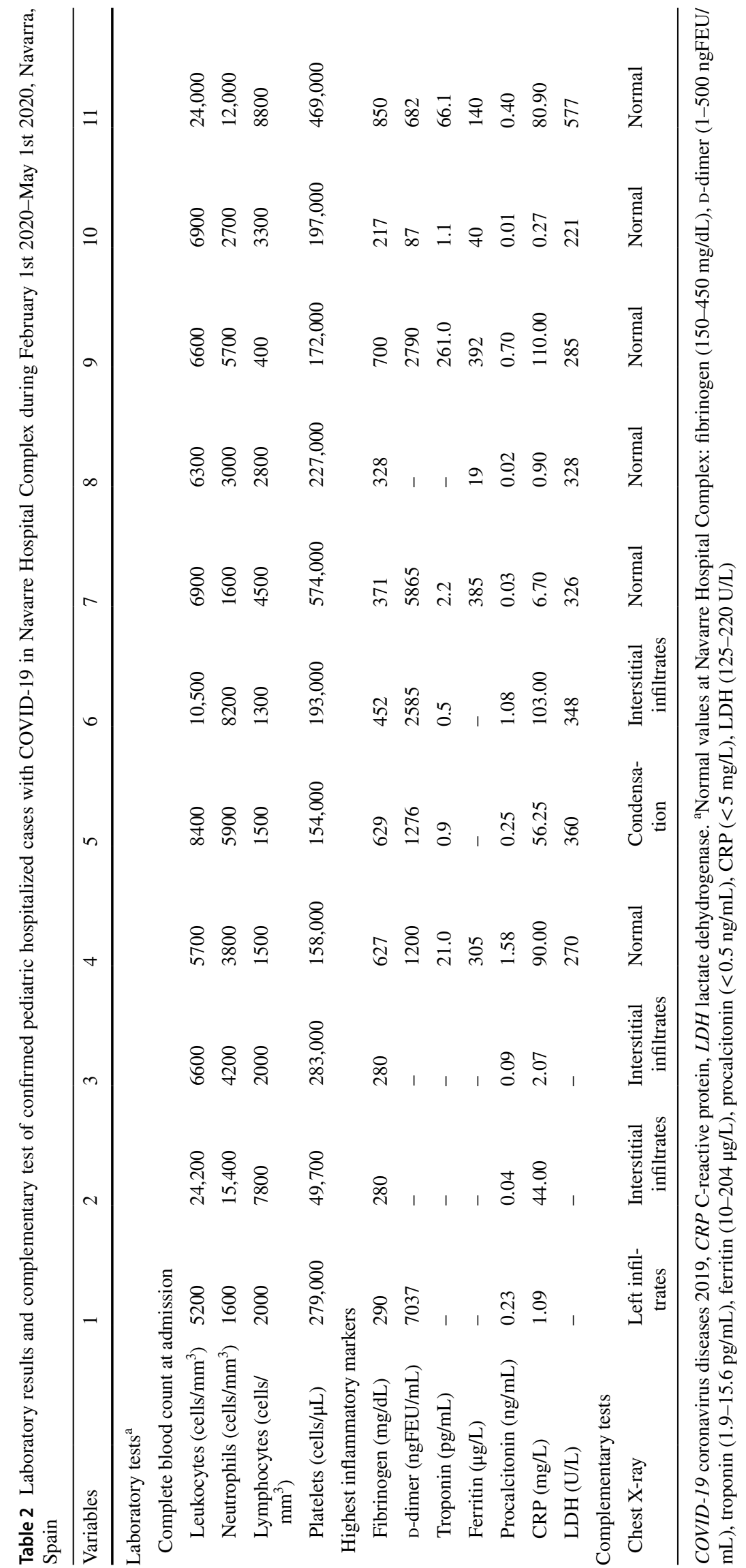




\section{Discussion}

This is the first report describing pediatric COVID-19 cases requiring hospitalization in Navarra, one of the 17 Autonomous Communities that constitute Spain. Given Navarra's well-developed health system, local characteristics, and size, all severe pediatric cases were treated in the capital's (Pamplona) tertiary hospitals. No pediatric cases were transferred outside the community during the first wave of the pandemic, making Navarra an ideal scenario for case-series reports.

Hospitalized cases were rare among children (hospitalization rate $1: 10,000$ ) with $180 \mathrm{CHN}$-hospitalized adults for each pediatric case and with an average hospital-stay of 6 days. From the 309 confirmed COVID-19 pediatric cases in Navarra, 11 (3.5\%) children needed hospital admission. Our results are consistent with previous data, showing that pediatric patients with COVID-19 generally have milder symptoms or are even asymptomatic of the disease. Our results also suggest that even though most children presented with mild-to-moderate symptoms and all children recovered in a short period of time, a very wide variety of clinical manifestations were observed. Symptoms were nonspecific, the classic COVID-19-triad (fever, cough and respiratory distress) was present in only one third of the cases, and fever and digestive symptoms were the most frequent complaints at admission. Blood test results were similar to those referred in adult patients [5, 6], especially in severe cases with frequent lymphopenia and increased inflammatory markers. Lymphopenia was more common in older patients. Chest X-ray was normal in more than half (6/11) of the cases. Atypical clinical presentations seen in children can make diagnosis more challenging, and exposure history and laboratory tests are keys to diagnosing pediatric patients.

In previous reports $[7,8,14]$, younger children and infants were more likely to have severe clinical manifestations than older children or teenagers; however, in our series, the most severe case appeared in a previously healthy 13-year-old boy. No significant differences were found between severe and non-severe cases, reflecting that children at any age can have severe complications related to SARS-CoV-2 infection. Following internal protocols, during the pandemic, all children with risk factors and suggestive COVID-19-symptoms were tested for SARS-CoV-2, but most hospitalized patients were previously healthy children. In most cases, mild symptoms were observed, but in some patients, the infection led to a serious disease. Tachycardia and refractive blood pressure were red flags for acute myocarditis, though risk factors for severe presentations remain unclear, and there is an urgent need for further research. The severe cardiac illness, also referred to as "Kawasaki-like", "septic-shock-like", or "pediatric-systemic-inflammatory response", needs to be better studied. The association between these cases and SARS-CoV-2 needs to be demonstrated. Case reports and global data recollection will be key to establishing the correct guidelines for the prevention, detection and treatment of this novel COVID-19 pediatric presentation.

Further investigation about the role that children play in viral transmission is urgent, as it will help to establish better social and public health policies. The proportion of asymptomatic cases among children makes it harder to identify pediatric cases, leading to high-risk situations when community transmission occurs. In our study, the route of transmission was mainly by contact with family members. No other sources, such as hospital-stay or travel to epidemic areas, were identified. Pediatric cases in our study appeared later in the quarantine period, and in many cases, children had close contact with a family member. Source contact, when known, was generally a relative (mostly parents $83 \%$ ). The period between parent's and children's diagnosis was in some cases very long, even longer than four weeks, which can be explained by different reasons. Patients with COVID-19 in Spain were told to do a home quarantine in an isolated room for 14 days after symptoms onset or discharge. The pediatric cases that appeared nearly a month after parent's diagnosis might indicate that either "the 14-day quarantine" was insufficient in length, leading to parents returning to normal family life while still being infectious, or that the home-isolation was performed incorrectly, leading to infection among house-hold members during quarantine. Other possible explanations might be that children's incubation period is longer than adults or that transmission occurred through another family member who acted as an asymptomatic carrier, or even outside the family cluster $[15,16]$. There is also known evidence of fecal shedding in the stool for several weeks [15-17] making it possible to get infected by a fecal-oral transmission among family members, especially among younger children. Prolonged viral shedding in patients who are symptomatic or are no longer symptomatic deserves further investigation. These data remain unclear. Our small sample size did not allow us to establish significant conclusions, and case-series reports and metaanalyses are needed to better understand SARS-CoV-2 infection in children. In order to control family clusters, epidemiological investigation and massive testing will be needed for the detection of hidden asymptomatic carriers.

Navarra is a wealthy community with a highly developed health system. All severe pediatric cases were referred to the main capital hospitals, and medical records were registered in a unified digital platform, making Navarra an ideal scenario for case-series reports. The main study limitation is that we only described hospitalized and confirmed COVID19 cases, which limited the sample size. An epidemiological 
study that includes all suspected, probable and non-hospitalized-COVID-19 cases should be performed in order to better understand the true epidemiology of this disease.

We have learned a lot about this virus and about the human response to infection in the past months; however, the effects on pediatric patients remain unclear and there is still much to learn about the real impact of SARS-CoV-2 on children, especially about the severe COVID-19 cases that have recently been reported. We hope that collaborative investigation, globalized case registers and local case-series reports, such as the one presented here, will help to better understand this new coronavirus.

Acknowledgements We would like to thank all the health workers from Navarra's Health Centers, for their enormous effort and excellent work in the management of the COVID-19 pandemic, especially the pediatricians from the CUN and CHN pediatrics departments. Most importantly, we thank the patients and their families for their patience, understanding and collaboration in this hard and difficult situation.

Author contributions MGL contributed to conception and design, analysis, and draft of the manuscript. UMM contributed to conception and design, acquisition and analysis, and draft of the manuscript. AEJ contributed to acquisition and critical revision of the manuscript. GHM contributed to design, acquisition, and draft of the manuscript. RGE contributed to design, acquisition, and critical revision of the manuscript. AAS contributed to conception and design, acquisition, and critical revision of the manuscript. AAV contributed to design, interpretation, and critical revision of the manuscript. HAM contributed to conception, analysis and interpretation, and critical revision of the manuscript. All authors have agreed to submit this article in the present form. Each has participated sufficiently in the conception and design of the work. All authors approved the final manuscript as submitted and agree to be accountable for all aspects of the work.

Funding No funding was secured for this study.

\section{Compliance with ethical standards}

Ethical approval The local ethics committee approved the study on May 2020: Ethics Committee of Navarre Hospital Complex (CHN: PI_2020/38: Epidemiological study of respiratory infections caused by the new coronavirus SARS-COV-2 in the pediatric population).

Conflict of interest All authors declare that they have no conflicts of interests related to the publication of this study.

\section{References}

1. Wang C, Horby PW, Hayden FG, Gao GF. A novel coronavirus outbreak of global health concern. Lancet. 2020;395:470-3.

2. Zhu N, Zhang D, Wang W, Li X, Yang B, Song J, et al. A novel coronavirus from patients with pneumonia in China, 2019. N Engl J Med. 2020;382:727-33.
3. World Health Organization. Statement on the second meeting of the International Health Regulations (2005) Emergency Committee regarding the outbreak of novel coronavirus (2019nCoV). https://www.who.int/news-room/detail/30-01-2020-state ment-on-the-second-meeting-of-the-international-health-regul ations-(2005)-emergency-committee-regarding-the-outbreak-ofnovel-coronavirus-(2019-nCov). Accessed 30 Jan 2020.

4. WHO. Situation report number 102 , coronavirus disease. https:// www.who.int/emergencies/diseases/novel-coronavirus-2019/situa tion-report. Accessed 1 May 2020.

5. Chen N, Zhou M, Dong X, Qu J, Gong F, Han Y, et al. Epidemiological and clinical characteristics of 99 cases of 2019 novel coronavirus pneumonia in Wuhan, China: a descriptive study. Lancet. 2020;395:507-13.

6. Wu Z, McGoogan JM. Characteristics of and important lessons from the coronavirus disease 2019 (COVID-19) outbreak in China: summary of a report of 72314 cases from the Chinese Center for Disease Control and Prevention. JAMA. 2020;323:1239-42.

7. Castagnoli R, Votto M, Licari A, Brambilla I, Bruno R, Perlini S, et al. Severe acute respiratory syndrome coronavirus 2 (SARSCoV-2) infection in children and adolescents: a systematic review. JAMA Pediatr. 2020. https://doi.org/10.1001/jamapediat rics.2020.1467.

8. Chen ZM, Fu JF, Shu Q, Chen YH, Hua CZ, Li FB, et al. Diagnosis and treatment recommendations for pediatric respiratory infection caused by the 2019 novel coronavirus. World J Pediatr. 2020;16:240-6.

9. Dong Y, Mo X, Hu Y, Qi X, Jiang F, Jiang Z, et al. Epidemiological characteristics of 2143 pediatric patients with 2019 coronavirus disease in China. Pediatrics. 2020;145:e20200702.

10. Li Q, Guan X, Wu P, Wang X, Zhou L, Tong Y, et al. Early transmission dynamics in Wuhan, China, of novel coronavirus-infected pneumonia. N Engl J Med. 2020;382:1199-207.

11. Moreno-Galarraga L, Taveras EM. COVID-19 disease in children: not as mild as we have been led to believe. World J Pediatr. 2020;16:426-7.

12. Navarra Institute of Statistics. Navarra's government public health records. https://www.navarra.es/home_es/Gobierno+de+Navarra/ Organigrama/Los+departamentos/Economia $+y+H a c i e n d a / O r g a n$ igrama/Estructura+Organica/Instituto+Estadistica. Accessed 1 May 2020.

13. Chan JF, Yuan S, Kok KH, To KK, Chu H, Yang J, et al. A familial cluster of pneumonia associated with the 2019 novel coronavirus indicating person-to-person transmission: a study of a family cluster. Lancet. 2020;395:514-23.

14. Onder G, Rezza G, Brusaferro S. Case-fatality rate and characteristics of patients dying in relation to COVID-19 in Italy. JAMA. 2020. https://doi.org/10.1001/jama.2020.4683.

15. Wu Y, Guo C, Tang L, Hong Z, Zhou J, Dong X, et al. Prolonged presence of SARS-CoV-2 viral RNA in faecal samples. Lancet Gastroenterol Hepatol. 2020;5:434-5.

16. Qian G, Yang N, Ma AHY, Wang L, Li G, Chen X, et al. COVID19 transmission within a family cluster by presymptomatic infectors in China. Clin Infect Dis. 2020;71:861-2.

17. Xu Y, Li X, Zhu B, Liang H, Fang C, Yu G, et al. Characteristics of pediatric SARS-CoV-2 infection and potential evidence for persistent fecal viral shedding. Nat Med. 2020;26:502-5.

Publisher's Note Springer Nature remains neutral with regard to jurisdictional claims in published maps and institutional affiliations. 